\title{
Energy flow in photonic crystal waveguides
}

\section{Søndergaard, Thomas; Dridi, Kim}

\section{Published in:}

Physical Review B Condensed Matter

Link to article, DOI:

10.1103/PhysRevB.61.15688

Publication date:

2000

Document Version

Publisher's PDF, also known as Version of record

Link back to DTU Orbit

Citation (APA):

Søndergaard, T., \& Dridi, K. (2000). Energy flow in photonic crystal waveguides. Physical Review B Condensed Matter, 61(23), 15688-15696. https://doi.org/10.1103/PhysRevB.61.15688

\section{General rights}

Copyright and moral rights for the publications made accessible in the public portal are retained by the authors and/or other copyright owners and it is a condition of accessing publications that users recognise and abide by the legal requirements associated with these rights.

- Users may download and print one copy of any publication from the public portal for the purpose of private study or research.

- You may not further distribute the material or use it for any profit-making activity or commercial gain

- You may freely distribute the URL identifying the publication in the public portal

If you believe that this document breaches copyright please contact us providing details, and we will remove access to the work immediately and investigate your claim 


\title{
Energy flow in photonic crystal waveguides
}

\author{
T. Søndergaard* \\ Research Center COM, Technical University of Denmark, Building 345, DK-2800 Lyngby, Denmark
}

\author{
K. H. Dridi ${ }^{\dagger}$ \\ Optics and Fluid Dynamics Department, OFD-129, Risb National Laboratory, Frederiksborgvej 399, DK-4000 Roskilde, Denmark
}

(Received 5 January 2000)

\begin{abstract}
Theoretical and numerical investigations of energy flow in photonic crystal waveguides made of line defects and branching points are presented. It is shown that vortices of energy flow may occur, and the net energy flow along the line defect is described via the effective propagation velocity. Single-mode and multimode operations are studied, and dispersion relations are computed for different waveguide widths. Both strong positive, strong negative, and zero dispersion are possible. It is shown that geometric parameters such as the nature of the lattice, the line defect orientation, the defect width, and the branching-point geometry have a significant influence on the electrodynamics. These are important issues for the fabrication of photonic crystal structures.
\end{abstract}

\section{INTRODUCTION}

Photonic crystal structures exhibit efficient light-control properties. ${ }^{1-7}$ As optical technology permits higher transmission rates of information at higher speed with great capacity, many compact optical devices such as low-threshold lasers, microcavities, waveguides, and multiplexers based on photonic crystals have been proposed, ${ }^{8-10}$ in order to achieve miniaturization and exploit the excellent light confinement that they offer.

The main reason for the interest in photonic crystals is that their condensed matter physics allows the existence of photonic bandgaps. When a line defect is introduced in the perfect photonic crystal that exhibits a bandgap, waveguides in which guided modes are allowed may be created. Mode symmetries in some optical guides with two-dimensional photonic band-gap boundaries have been studied. ${ }^{11}$ In this context, periodic structures such as dielectric gratings that form a class of photonic crystals are known to exhibit high wavelength-dependent modal dispersion. Just as wavelength dispersion is an important characteristic of fibers and dielectric slab waveguides, a few fundamental issues regarding the properties of waveguides with photonic crystal claddings have to be clarified. Figure 1 shows the energy flow (Poynting vector) in a conventional dielectric slab waveguide and in a waveguide with a photonic crystal cladding with a triangular lattice of GaAs rods in air, computed with the use of the finite-difference time-domain (FDTD) method. ${ }^{12}$ In conventional planar slab waveguides, light is guided in the high index film layer in which plane-wave components propagate via the mechanism of total internal reflection at planar material interfaces. In these waveguides, a Gaussian distribution of energy is present with a tail continuing in the cladding, and there is a net effective energy flow with the Poynting vector directed in the direction along the guide. On the other hand, photonic crystal waveguides may have a better confinement of energy in the guiding section because of the photonic band-gap phenomenon that prohibits radiation of energy in the cladding. In these guides, vortices may appear in the Poynting vector plot. The periodic lattice presents a photonic potential that restricts electromagnetic energy to the line-defect region. These vortices of energy flow show that diffraction and reflection processes at the defect-cladding interface force some wave components to be delayed and reflected in the opposite direction of net energy flow, but as guided modes conserve their form and positive net energy flow occurs, the reflected fields must add up in phase without significant distortion of the forward traveling field, propagating in a waveguide that can be regarded as a series of resonant cavities whose modes are matched.

Investigations of the time-average energy flow (Poynting vector), the effective velocity of energy transport along the waveguide, the single-mode/multimode operation, and the dispersion relations as a function of the width and orientation of the waveguide are therefore important issues. In this paper, theoretical and numerical investigations on the fundamental electrodynamics of two-dimensional waveguides with photonic crystal claddings are presented, focusing mainly on claddings with triangular and quadratic lattices of GaAs rods in a background of air. Recently, an investigation of twodimensional cladding structures of finite height was given in Ref. 13.

Furthermore, a study of energy flow in photonic crystal waveguides with sharp bends and a discussion of the electrodynamics of branching points are presented. In such branching points, as in constrictions and bends, bound states can occur, ${ }^{14}$ and resonant modes must be matched to the waveguide modes in order to obtain high transmission coefficients $^{14-17}$ with efficient redistribution of energy.

In Sec. II the method of analysis used in the calculation of band diagrams is presented. Analyses of straight photonic crystal waveguides are discussed in Sec. III, where two line defect orientations are studied for photonic crystal waveguides with triangular lattices. In Sec. IV, sharp bends and branching points are discussed, and geometric influences on the energy flow are underlined. We conclude in Sec. V.

\section{METHOD OF ANALYSIS}

The analyses in this paper rely on two numerical models based on the FDTD method ${ }^{12}$ and Bloch theory, respectively, for the TM polarization (the electric vector field is oriented 


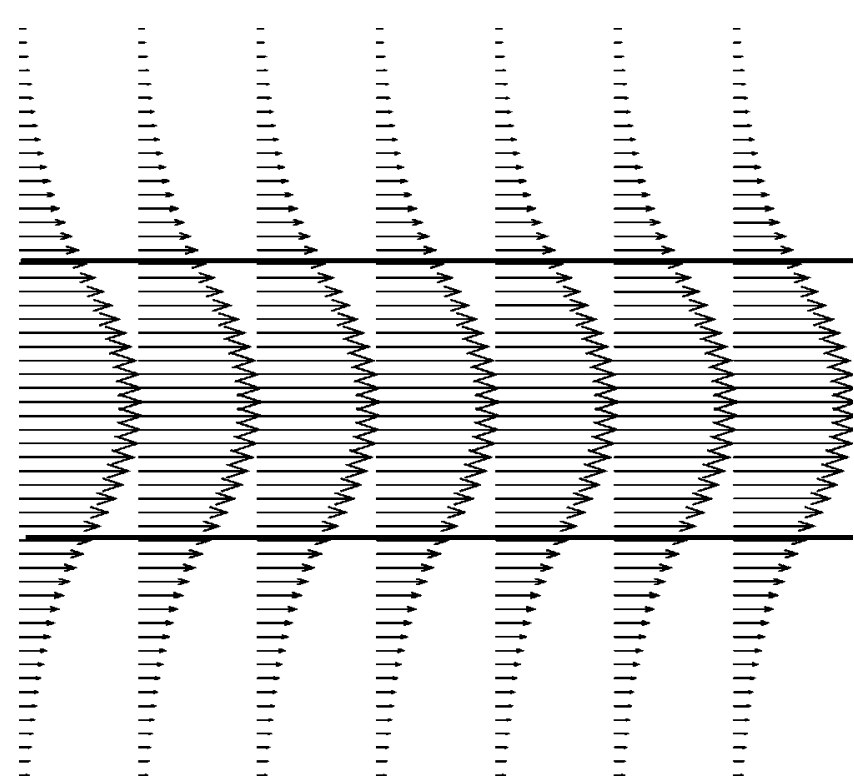

(a)

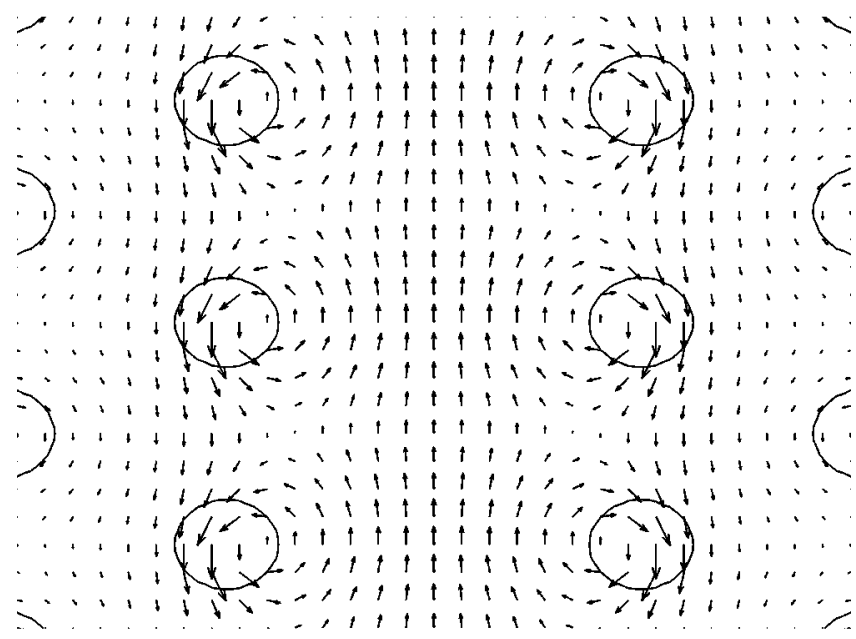

(b)

FIG. 1. Energy flow (Poynting vector) in a conventional symmetric dielectric slab waveguide and in a waveguide with a photonic crystal cladding with a triangular lattice of GaAs rods in air.

along the axis of the rods). The Bloch theory model is based on plane-wave expansions and a variational principle. ${ }^{18-20}$ The energy flow, i.e., the Poynting vector and the energy propagation velocity, is analyzed for waveguides based on photonic crystal claddings.

We choose a two-dimensional photonic crystal with circular dielectric rods, of dielectric constant $\varepsilon=11.4$ (the permittivity of GaAs at optical frequencies), arranged on a triangular lattice in a background of air with $\varepsilon=1$.

The photonic crystal is characterized by discrete translational symmetry in the $x y$ plane (see Fig. 2) and is fully described by the unit cell shown in Fig. 2(a). This structure exhibits a bandgap for certain values of rod diameter $D$ and lattice constant $\Lambda$, i.e., there is a frequency interval, a TM photonic band gap, where TM polarized modes are not allowed to propagate in the $x y$-plane. However, by introducing a line defect in the crystal, as shown in Fig. 2(b), TM polarized modes with a frequency within the band gap of the perfect photonic crystal may exist. These modes are local-

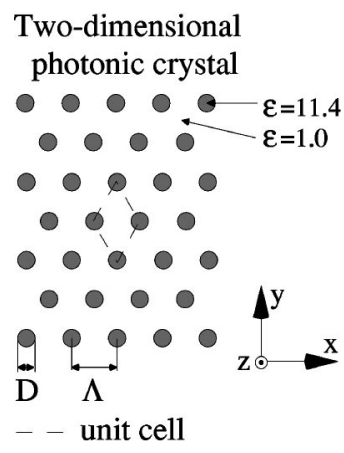

(a)

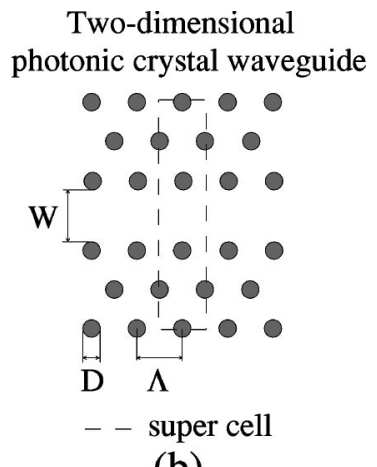

(b)
FIG. 2. (a) Two-dimensional photonic crystal with circular dielectric rods arranged on a triangular lattice in a background of air. The simplest unit cell required for the representation of the structure is shown with a dashed line. (b) Two-dimensional photonic crystal waveguide made by introducing a line defect in the photonic crystal. The width of the waveguide is denoted by $W$. A supercell is shown with a dashed line.

ized to the region of the line defect. A way of modeling straight waveguides, such as the one shown in Fig. 2(b), is to approximate the waveguide by a periodic structure using a supercell approximation. The superperiodicity is obtained by spatially repeating the supercell, which is the period of the discrete translational representation [see Fig. 2(b)]. Due to this approximation, the considered structure is a periodic array of waveguides, where the separation between the waveguides is determined by the height of the supercell. To ensure no coupling of energy between guided modes in neighboring waveguides, the height of the supercell is increased to an appropriate level.

The photonic crystal and the periodic approximation of photonic crystal waveguides are both characterized by discrete translational symmetry. According to Bloch's theorem the electric field and the magnetic field in discrete translationally symmetric structures may be written as an expansion of Bloch modes. The magnetic field is expressed as

$$
\mathbf{H}_{\mathbf{k}, n}(\mathbf{r})=\sum_{\mathbf{G}} \sum_{\nu=1,2} \mathbf{h}_{\mathbf{k}, \mathbf{G}, \nu, n} e^{i(\mathbf{k}+\mathbf{G}) \cdot \mathbf{r}},
$$

where $\mathbf{k}$ is the Bloch wave vector, $\mathbf{G}$ is a reciprocal lattice vector, and $\nu$ represents the two field directions perpendicular to $\mathbf{k}+\mathbf{G}$ (ensuring transversality, i.e., $\nabla \cdot \mathbf{H}=0$ ). The index $n$ is the band number. The frequency of the Bloch mode is denoted $\omega_{\mathbf{k}, n}$. The magnetic field calculation relies on a variational method based on minimization of the functional

$$
E(\mathbf{H})=\frac{\left\langle\nabla \times\left(\left(1 / \varepsilon_{r}(\mathbf{r})\right) \nabla \times \mathbf{H}\right) \mid \mathbf{H}\right\rangle}{\langle\mathbf{H} \mid \mathbf{H}\rangle} .
$$

When this functional is at a minimum, the $\operatorname{argument} \mathbf{H}$ is a solution to the wave equation

$$
\nabla \times\left(\frac{1}{\varepsilon_{r}(\mathbf{r})} \nabla \times \mathbf{H}(\mathbf{r})\right)=\frac{\omega^{2}}{c^{2}} \mathbf{H}(\mathbf{r}) .
$$

By applying a trial vector on the form (1) in Eq. (2), the functional effectively becomes a function of the coefficients $\mathbf{h}_{\mathbf{k}, \mathbf{G}, \nu, n}$, and the problem is reduced to varying the coeffi- 


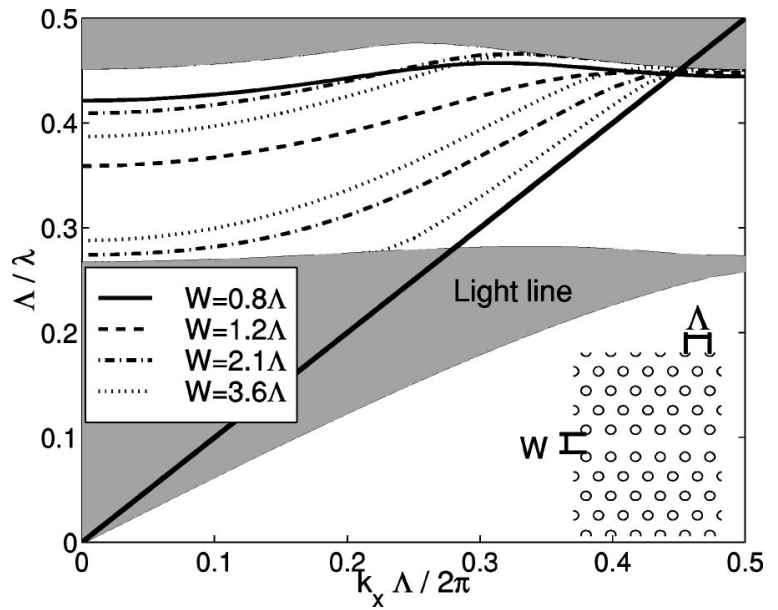

FIG. 3. Allowed frequencies for TM polarized modes in the straight photonic crystal waveguide (shown as an inset) for four different values of the width $W$.

cients along a path that minimizes the functional. An efficient iterative approach that performs this task is described in Ref. 19. Higher-order solutions are found by restricting the trial vectors to be orthogonal to all previously found eigenvectors, using the same minimization principle. For more details on the numerical method see Refs. 18 and 20. We choose the rod diameter $D=0.4 \Lambda$ corresponding to the largest TM photonic band-gap ${ }^{2}$ for the following investigations in order to exploit the band-gap effect.

\section{STRAIGHT PHOTONIC CRYSTAL WAVEGUIDES}

\section{A. Waveguides with a triangular lattice cladding with first orientation}

Figure 3 shows a TM band diagram for the twodimensional photonic crystal waveguide for four values of the width $W . \Lambda / \lambda$ is the normalized frequency, where $\lambda$ is the free-space wavelength, and $k_{x} \Lambda / 2 \pi$ is the component of the normalized wave vector along the line defect. The gray regions in the figure represent a continuum of TM modes of specific frequencies and specific values of the $x$ component of the Bloch wave vector allowed to propagate in the twodimensional photonic crystal cladding. A TM band gap exists for the photonic crystal cladding in the interval from $\Lambda / \lambda \approx 0.27$ to 0.45 where no cladding modes are allowed. However, as a line defect is introduced in the perfect crystal, one or more defect bands may appear within the photonic band gap. Figure 3 shows that only one defect band exists for the widths $W=0.8 \Lambda$ and $W=1.2 \Lambda$. As the width of the waveguide is increased to $W=2.1 \Lambda$, two defect bands are allowed, and for $W=3.6 \Lambda$ three defect bands appear. Defect modes appear to break away from the continuum of modes at the top of the cladding band gap, having small bandwidths (few frequencies allowed in one defect band), and they appear to "descend" toward the bottom of the cladding bandgap, gaining a larger bandwidth as $W$ is increased. As the width of the waveguide increases, more defect modes see their individual dispersion relation approach that of freespace propagating modes given by the light line $\Lambda / \lambda$ $=k_{x} \Lambda / 2 \pi$. This is due to the fact that more modes are allowed to propagate in a broader line defect region with cer-

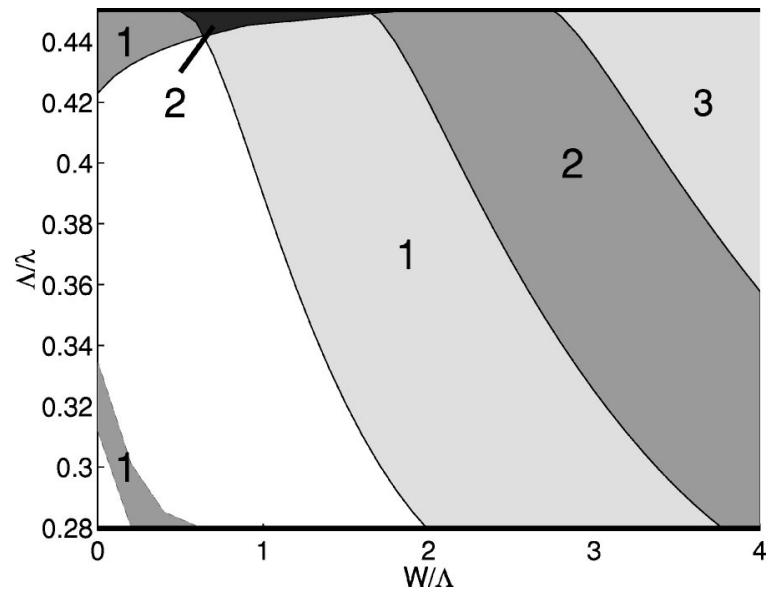

FIG. 4. Number of guided modes in the straight triangularlattice photonic crystal waveguide (first orientation) as a function of the waveguide width and the frequency.

tain wave vectors in such a way that these guided modes weakly sense the effect of the waveguide width. Although three defect bands exist for the waveguide of width $W$ $=3.6 \Lambda$, there is a frequency interval where only one of the defect modes is allowed, and consequently single-mode guidance of light is possible for frequencies within this interval $(\Lambda / \lambda<0.29)$. A similar observation is valid for $W$ $=2.1 \Lambda$, for which single-mode operation is possible for $\Lambda / \lambda<0.38$ although two defect bands are present within the cladding band gap.

A clearer picture of the single-mode/multimode behavior can be obtained by considering the results of calculations of the number of localized modes in the line defect as a function of the waveguide width and the frequency (see Fig. 4). The number assigned to different areas in the $(\Lambda / \lambda, W / \Lambda)$ plot represent the number of guided modes as a function of $W / \Lambda$ values between 0 and 4 , and as a function of the normalized frequency in the band gap of the cladding. Zero up to three guided modes are observed. For widths larger than approximately $3.8 \Lambda$, there are no frequency intervals within which only one defect mode is guided by the line defect. As a rule of thumb, single-moded guidance appears to be possible for waveguide widths being on the order of, or smaller than, a free-space wavelength. The white area represents a region in the band gap of the cladding where no guided modes are allowed. Filtering of certain normalized frequencies within the band gap of the cladding is possible for $W / \Lambda<2$, especially for narrow waveguides with $W / \Lambda \approx 0.8$ where only one defect band is present, and where this band has a small bandwidth for which all $k_{x} \Lambda / 2 \pi$ are allowed (see Fig. 3). Note that the domain with two guided modes for $W<1.8 \Lambda$ is not due to two separate defect bands overlapping in frequency. It is the same defect mode that is allowed to propagate with two different values of $k_{x} \Lambda / 2 \pi$. This is illustrated by considering the defect band for $W=0.8 \Lambda$ in Fig. 3, where there may be two allowed values for $k_{x} \Lambda / 2 \pi$ for the same frequency $\Lambda / \lambda$.

Considering the defect band corresponding to $W=1.2 \Lambda$ in Fig. 3 , modes corresponding to different $\left(\Lambda / \lambda, k_{x} \Lambda / 2 \pi\right)$ solutions on this defect band may behave quite differently in terms of their flow of energy in the waveguide. Figure 5 shows the real part of the complex Poynting vector, $\mathbf{P}=\mathbf{E}$ 


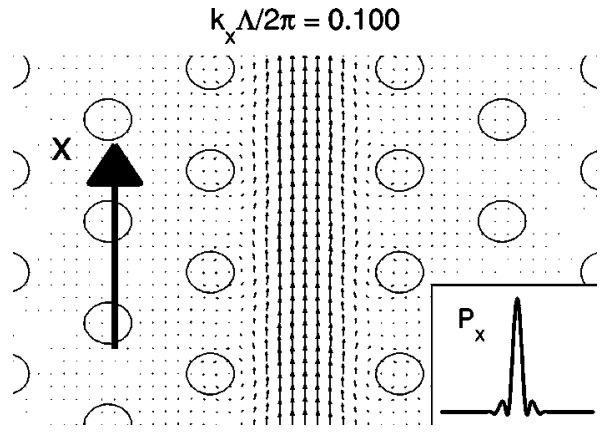

FIG. 5. The real part of the complex Poynting vector $\mathbf{P}=\mathbf{E}$ $\times \mathbf{H}^{*}$ for a Bloch mode, where the $x$ component of the Bloch wave vector $k_{x}$ is given by $k_{x} \Lambda / 2 \pi=0.100$. The inset shows an example of the $x$ component of the Poynting vector across the waveguide.

$\times \mathbf{H}^{*}$, for $k_{x} \Lambda / 2 \pi=0.100$, with $E=\left(1 / i \omega \varepsilon_{r}(\mathbf{r}) \varepsilon_{0}\right) \nabla \times \mathbf{H}, i$ $=\sqrt{-1}$, where $*$ denotes complex conjugation, and $\varepsilon_{0}$ is the dielectric constant in vacuum. The inset shows the $x$ component of the Poynting vector. The figure shows that the energy flows primarily in the $\hat{\mathbf{x}}$ direction. However, the picture changes dramatically when $k_{x} \Lambda / 2 \pi$ is chosen closer to 0.5 . For both cases $k_{x} \Lambda / 2 \pi=0$ and $k_{x} \Lambda / 2 \pi=0.5$, no energy is allowed to flow, and in these two limits, light is not guided. The situation for $k_{x} \Lambda / 2 \pi=0.450$ is illustrated in Fig. 6 . The inset shows the $x$ component of the Poynting vector across the waveguide. The figure indicates that energy is no longer flowing primarily in the $\hat{\mathbf{x}}$ direction, and vortices of energy flow appear as turbulence. In this case energy moves circularly near the dielectric rods closest to the waveguiding region, and energy appears to be coupled backwards. The net energy flow is practically zero across the waveguide section. The situation where $k_{x} \Lambda / 2 \pi=0.5$ appears to be a resonant state where two-dimensional standing waves are possible, and the waveguide might be considered as a series of resonant cavities.

For $W=3.6 \Lambda$ there are three defect bands (see Fig. 3). Poynting vector plots for three defect modes belonging to three different defect bands are shown in Fig. 7 for low values of $k_{x} \Lambda / 2 \pi$ on each individual defect band. For all three cases the energy flows primarily in the $\hat{\mathbf{x}}$ direction. The profiles indicate that the pattern of the modes resembles that of standing waves along the $y$ axis, which is what could have been expected for modes guided between two parallel metal-

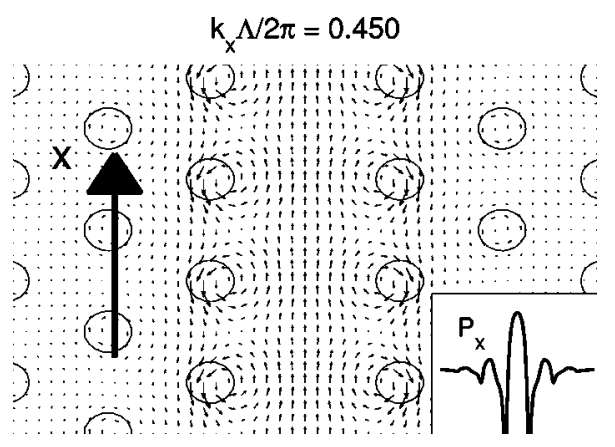

FIG. 6. The real part of the complex Poynting vector $\mathbf{P}=\mathbf{E}$ $\times \mathbf{H}^{*}$ for a Bloch mode, where the $x$ component of the Bloch wave vector $k_{x}$ is given by $k_{x} \Lambda / 2 \pi=0.450$. The inset shows an example of the $x$ component of the Poynting vector across the waveguide.

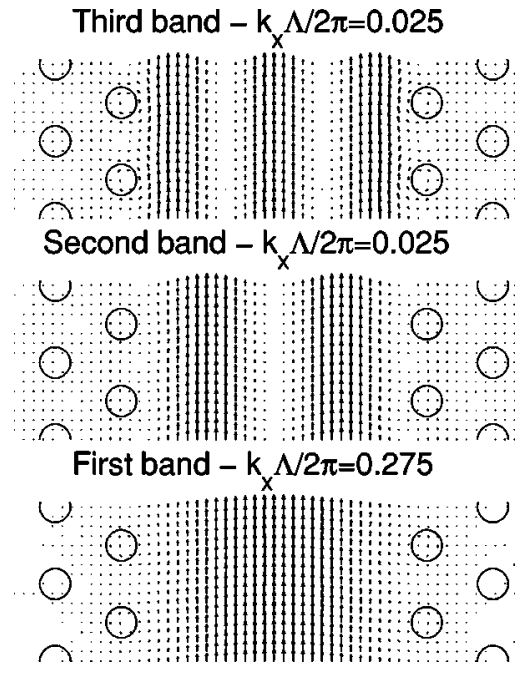

FIG. 7. The real part of the complex Poynting vector $\mathbf{P}=\mathbf{E}$ $\times \mathbf{H}^{*}$ for the first-order, second-order, and third-order guided modes in the photonic crystal waveguide. $W=3.6 \Lambda$.

lic plates. In this case the photonic crystal material on either side of the waveguide acts similarly to metallic reflectors for microwave frequencies. A key difference between the photonic crystal waveguide and a metallic-parallel-plate waveguide is that whereas a metallic-parallel-plate waveguide has a lower cutoff frequency, a photonic crystal line defect waveguide may have both a lower and a higher cutoff frequency. This difference means that in photonic crystal line defect waveguides both strong positive and strong negative dispersion are possible.

The effective energy propagation velocity $V_{E}$ may be determined as the time average net energy flow in the $\hat{\mathbf{x}}$ direction through a line parallel to the $y$ axis divided by the time and space average energy storage: ${ }^{21}$

$$
\frac{V_{E}}{c}=\frac{\int_{y=-\infty}^{+\infty} P_{x} d y}{1 / \Lambda \int_{y=-\infty}^{+\infty} \int_{x=0}^{\Lambda} \varepsilon_{0} \varepsilon_{r}(\mathbf{r})|\mathbf{E}|^{2}+\mu_{0}|\mathbf{H}|^{2} d x d y} .
$$

Note that $\int_{-\infty}^{+\infty} P_{x}(x, y) d y$ is independent of $x$ (which has been checked numerically). A calculation of this velocity is shown in Fig. 8 for two choices of the waveguide width $W$. The circles and crosses on the smooth curves show results for the flow velocity obtained using Eq. (4), whereas the smooth curves were obtained by calculating $d \omega / d k_{x}$, i.e., the smooth curves may be obtained directly from the dispersion relations shown in Fig. 3. Indeed the effective flow velocity equation (4) equals the group velocity $\left(d \omega / d k_{x}\right)$ in the case of a perfect dielectric. The narrow waveguide with $W$ $=1.2 \Lambda$ has only one defect band (see Fig. 3). Its band frequency edges are seen at the two edges of the dashed curve, where the energy propagation velocity drops to zero. From the lower band edge the velocity increases with frequency, whereas the velocity decreases with frequency closer to the higher band edge. Consequently, both strong positive and negative dispersion are possible. Furthermore, there is a frequency range where the slope of the dashed curve is negligible, indicating that zero dispersion is possible around the 


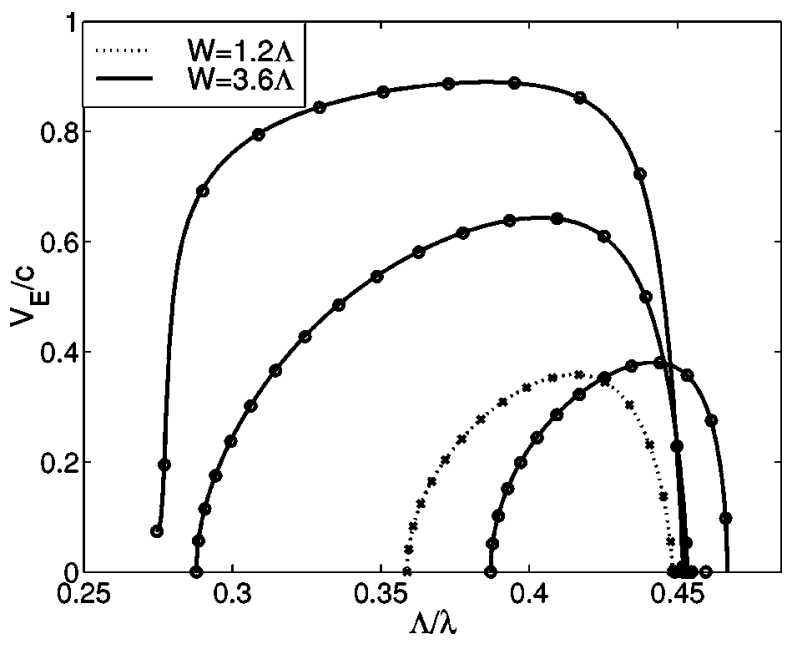

FIG. 8. Energy propagation velocity for guided modes in the two-dimensional photonic crystal waveguide for two choices of the waveguide width $W$. The smooth curves were obtained directly from the dispersion relations by calculating $d \omega / d k_{x}$, whereas the discrete points were obtained using Eq. (4).

center frequency with the maximal effective flow velocity $V_{E} / c \approx 0.36$ for this defect band. The wider waveguide with $W=3.6 \Lambda$ has three defect bands (see Fig. 3). The third-order band $(0.39<\Lambda / \lambda<0.465)$ is breaking away from the continuum of cladding modes (from higher frequencies to lower ones) with a maximal effective flow velocity of $V_{E} / c$ $\approx 0.38$ for this band in the band gap of the cladding (this third-order defect band does not have all its spectrum inside the band gap ). The second-order defect band $(0.29<\Lambda / \lambda$ $<0.45$ ) is wider and is inside the band gap of the cladding. More modes have small dispersion in this band than what was the case for the third-order band. The maximal effective flow velocity for this band is $V_{E} / c \approx 0.63$. The first-order band has a dispersion relation that approaches that of freespace propagation. It is the widest of the three defect bands for $W=3.6 \Lambda$, and more modes have small dispersion compared to the higher-order bands. The maximal effective flow velocity for the first-order band is $V_{E} / c \approx 0.87$. As the width of the waveguide is increased, defect bands break free from the continuum of cladding modes at the top of the band gap of the cladding, and move into the band gap, widening their bandwidth. Their modes obtain higher $V_{E} / c$ values, and the defect band's $V_{E} / c$ curves becomes more and more flat for center frequencies of the defect bands as $W / \Lambda$ is increased.

\section{B. Waveguides with a triangular lattice cladding with second orientation}

Figure 9 shows the band diagram for the actual photonic crystal waveguide with a line-defect orientation perpendicular to the previous one ( $\hat{\mathbf{x}}$ is redefined to be the direction along the line defect for this second orientation). The allowed frequencies are computed for TM polarized modes in the straight photonic crystal waveguide (shown as an inset) for three different values of the width $W$. The dispersion relations are in this case quite different from those of the previous line-defect orientation (see Fig. 3). It is important to note that the line defect with the orientation of Fig. 9 has a cladding boundary with a $\sqrt{3} \Lambda$ periodicity, whereas the line

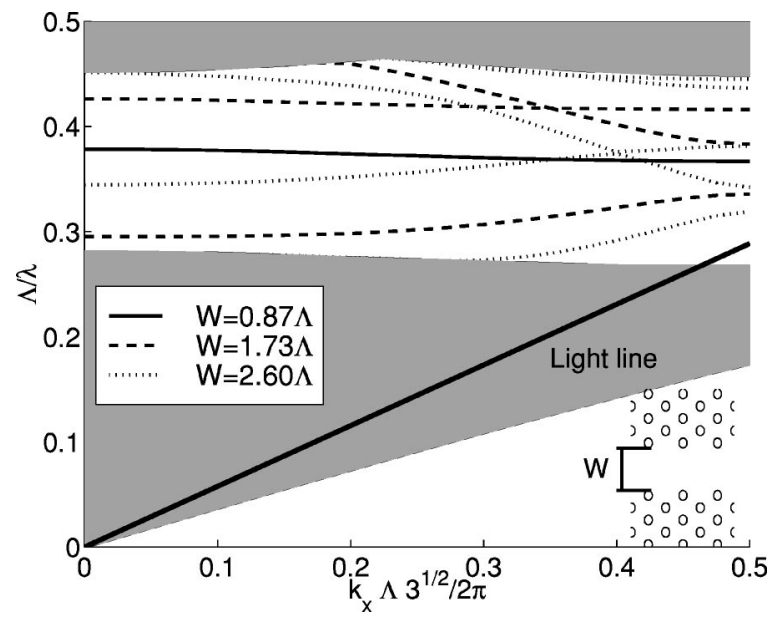

FIG. 9. Allowed frequencies for TM polarized modes in the straight photonic crystal waveguide (shown as an inset) for three different values of the width $W$.

defect with the orientation of Fig. 3 has a cladding boundary with a $\Lambda$ periodicity. Due to this difference in periodicity the light line is positioned almost entirely below the band gap in the case of the second orientation.

From Fig. 9 we observe that only one defect band exists for $W=0.87 \Lambda$, and it appears to be quite flat with a narrow bandwidth. For $W=1.73 \Lambda$ and $W=2.6 \Lambda$, three and five defect bands are allowed, respectively. As $W$ is increased, defect bands appear to break away from the upper cladding band gap edge at the right, gaining in bandwidth until they detach themselves completely from the upper cladding band gap edge at the left ( contrary to the line defects of Fig. 3), the normalized frequency of the defect band being a descending function of $k_{x} \sqrt{3} \Lambda / 2 \pi$. The defect bands subsequently have their bandwidths reduced as $W$ is increased further, and they appear flat as they reach the middle of the cladding band gap. As the defect width increases further, the bandwidth of the defect bands increases once again, and the normalized frequency of the defect band becomes an ascending function of $k_{x} \sqrt{3} \Lambda / 2 \pi$, with defect bands finally reaching the lower edge of the cladding band gap on the left side of Fig. 9. Both negative and positive dispersion are also achievable with this type of waveguide. Note that for the second orientation also the defect bands converge toward the light line as the width $W$ increases. The large difference between defect band dispersion relations for the two waveguide orientations can be attributed to the different positions of the light line relative to the band gap.

Figure 10 shows the number of guided modes in the straight photonic crystal waveguide with the line-defect orientation of Fig. 9 as a function of the waveguide width and the frequency. A comparison with the previous orientation (see Fig. 4) again shows a completely different distribution of guided modes. Single-mode operation seems possible for $W / \Lambda>4$. Figure 10 shows that there are band gaps for the guided modes in the band gap of the cladding. These gaps are seen as white areas where no guided mode is allowed. These gaps are surrounded by many discrete areas of singlemode operation. The number of guided modes is highly dependent on the width of the waveguide, and there is a more complicated distribution of guided modes for this second line defect orientation. 


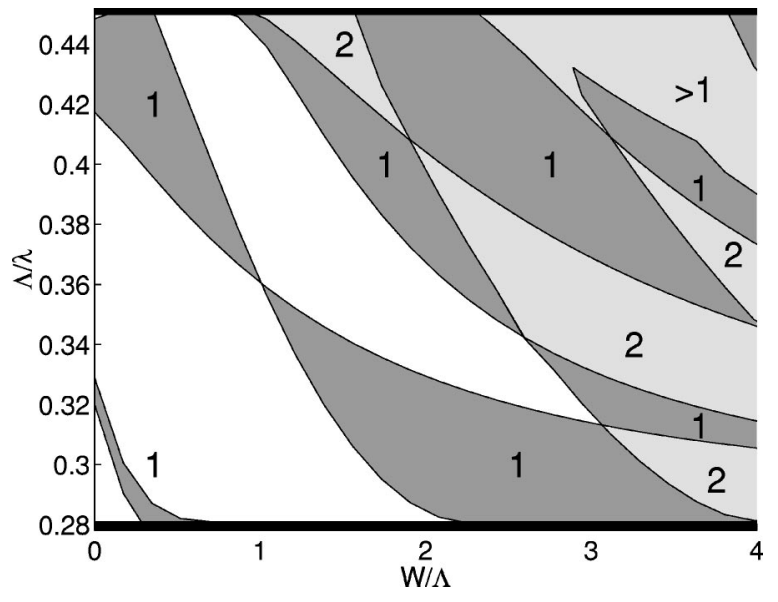

FIG. 10. Number of guided modes in the straight triangularlattice photonic crystal waveguide (second orientation) as a function of the waveguide width and the frequency.

The dispersion relations of the photonic crystal waveguides of Fig. 9 differ significantly from those of Fig. 3, indicating that the characteristics of photonic crystal waveguides are highly dependent on the orientation of the line defect. Operations of dispersion compensation, sensing, and filtering could be performed by such photonic crystal structures of definite length.

\section{BRANCHING POINTS IN PHOTONIC CRYSTAL WAVEGUIDES}

The following analysis relies on a numerical model based on the FDTD method, where a collimated beam is used as a source of excitation. Figure 11 shows a quadratic $\left(90^{\circ}\right.$ symmetry) and a triangular ( $60^{\circ}$ symmetry) lattice crystal with some of the most obvious configurations of waveguide connections (branching points) for the redistribution of energy. Each branching point geometry is specifically marked $\left(q_{1}\right.$ $-q_{4}$ and $\left.t_{1}-t_{7}\right)$. Due to the $60^{\circ}$ symmetry, the triangular lattice offers more natural branching configurations. With reference to Fig. 11, the branching points in the following investigations are illuminated from the left to the right through the horizontal waveguide section. The remarkable ability of photonic crystal waveguides to guide light around sharp corners with radii of curvature that are in the subwave-

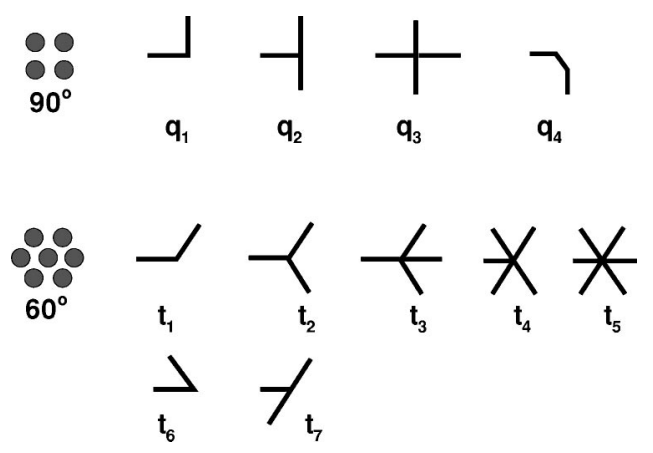

FIG. 11. Quadratic ( $90^{\circ}$ symmetry) and triangular ( $60^{\circ}$ symmetry) lattice crystals with some of the most obvious configurations of waveguide connections (branching points) for the redistribution of energy. length scale has been demonstrated for quadratic lattice claddings in Ref. 16. Contrary to conventional planar slabwaveguides, light can be guided around very sharp bends without significant reflections in the opposite direction of the incident energyflow and without radiation losses thanks to the band gap effect. With the $q_{4}$ geometry (see Fig. 11), it was shown that the transmission coefficient of the $q_{1}$ configuration could be improved in order to obtain coefficients above $98 \%$ (theoretically) when the dispersion relation of the branching region, a (11) waveguide section in the case of Ref. 16, does not differ significantly from those of the connecting waveguides, indicating the significance of matching the width of the connecting waveguides and the geometry of the region of the transition from the linedefect to the cladding.

Figure 12 shows the electric field in two branching points, $q_{2}$ and $q_{3}$, for the photonic crystal waveguide with a quadratic lattice. For waveguide widths of $W=1.6 \Lambda$, guidance of light is possible for the band of normalized frequencies in the range from $\Lambda / \lambda=0.305$ to $\Lambda / \lambda=0.435$. The $q_{3}$ configuration does not have power transmission coefficients in the horizontal direction above $18.28 \%$ around the center frequency of this band. The steady-state calculation of transmission coefficients takes into account the effects of reflections from outside the photonic crystal. Each of the two vertical waveguides carry about $38.48 \%$ of the incident power, and there is about $5 \%$ of the incident power that is reflected in the horizontal direction. Figure 13 shows a Poynting vector plot for this case. The difference in power coupling is due to the geometry of the branching point where radiation in the vertical direction is favored because the vertical waveguides are illuminated before the horizontal waveguide. In order to obtain a more equal power splitting, the entrance of each vertical waveguide might be changed. This could be done by introducing an extra rod that is centered about the symmetry axis of the vertical waveguides with a rod radius that is smaller than that of the waveguide cladding. In general, the optimal design of branching sections must result in geometries that match the resonant cavity modes of the branching point to the waveguide modes. ${ }^{17}$ The symmetry of the cavity modes must match the symmetry of all waveguide modes. Furthermore, the width of the waveguides must also be chosen carefully in order to avoid backward reflections due to mismatch of the dispersion relations of all the waveguides connected to the branching point. The photonic crystal waveguide with the triangular lattice cladding offers many options for the redistribution of energy into multiple waveguides. Figure 14 shows single channel waveguide-transmission coefficients for the $t_{2}$ and $t_{3}$ type of branching points with triangular lattices for $W=1.332 \Lambda$ for three normalized frequencies in the defect band. The fractions of incident power that are coupled into the oblique channels above and below the horizontal waveguide are equal because of symmetry. The data represented by squares are for a single oblique channel waveguide in the $t_{2}$ case. Diamonds represent data for one of the oblique channels in the $t_{3}$ case, and left arrows represent data for the horizontal channel waveguide in the $t_{3}$ case. The $t_{2}$ configuration has excellent transmission properties for all three normalized frequencies in the defect band, with a total transmission coefficient above 0.98 for the center frequencies of the defect band. In the $t_{3}$ case, a more pro- 


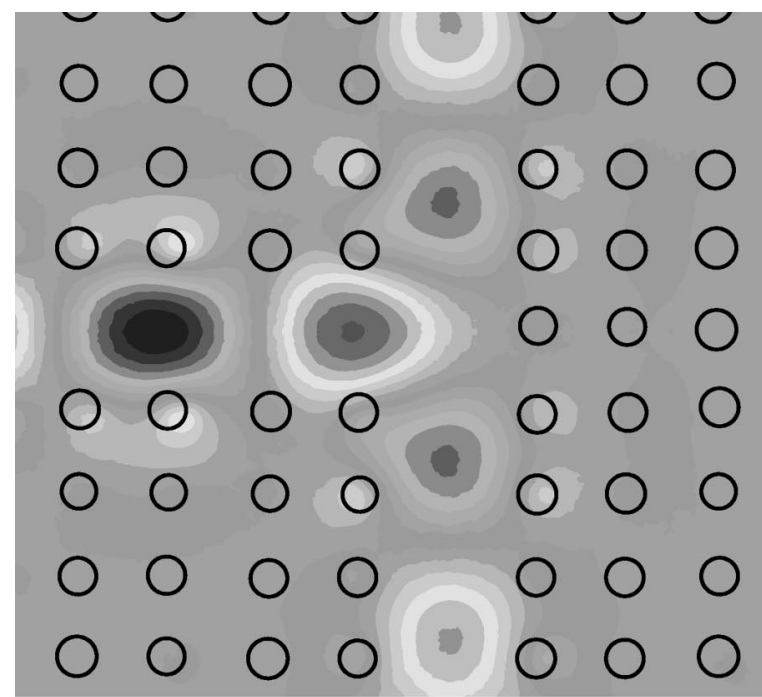

(a)

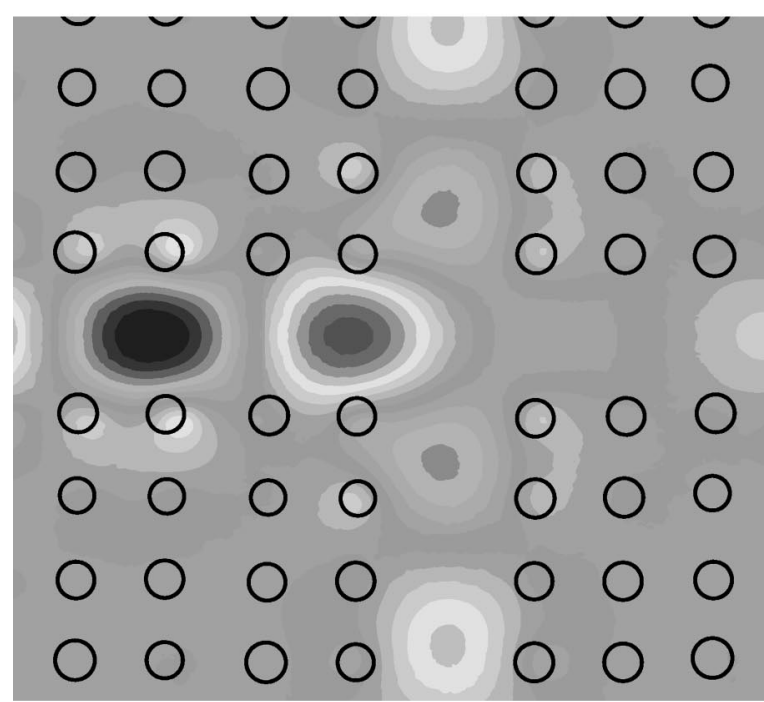

(b)

FIG. 12. Electric field in two branching points, $q_{2}$ and $q_{3}$, for the photonic crystal waveguide with a quadratic lattice.

nounced difference in the power transmitted in the horizontal direction is observed for the different frequencies, whereas a less significant difference is seen for the power transmitted in the oblique direction. A third of the incident power is transmitted horizontally in the $t_{3}$ case for a normalized frequency, $\Lambda / \lambda$, of 0.4 , whereas only about $10 \%$ of the power is carried away in the oblique waveguide. The horizontal waveguide after the branching point carries about three times as much power as the oblique waveguide for $\Lambda / \lambda=0.4$. This asymmetric coupling of energy is diminished for frequencies away from the center frequencies. Figure 15 shows the energy flow (Poynting vector) in branching points of type $t_{2}$ and $t_{3}$ for a triangular lattice with $\Lambda / \lambda=0.4$ and $W$ $=1.332 \Lambda$. The $t_{2}$ configuration ensures a highly symmetric power coupling with low-backward reflections. As photons approach the branching point cavity, the photonic potential diminishes because photons experience a decreasing photonic crystal wall, and tunneling occurs. A turbulent vortex of energy flow appears in the cladding region near the corner of the branching point. This gives the photons momentum as

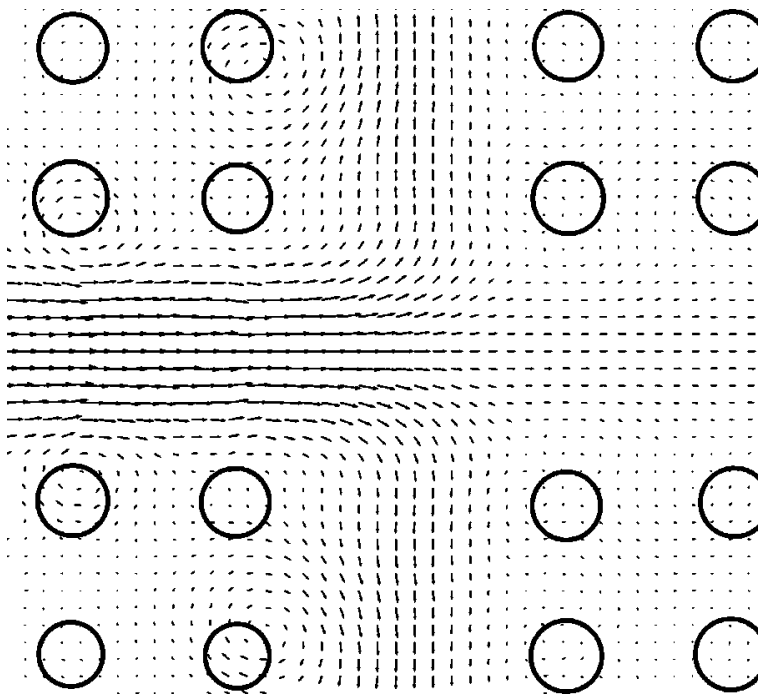

FIG. 13. Energy flow in a branching point of type $q_{3}$ for a quadratic lattice.

they approach the cavity, and the high-photonic potential in the horizontal direction forces electromagnetic energy to be redirected in the oblique directions. In the $t_{3}$ configuration, however, there is no forbidden energy gap for the incident modes along the horizontal direction in the defect band. The asymmetric coupling occurs because the dispersion relations are unchanged in the horizontal direction, and incident wavevectors do not allow a proper coupling of energy into the modes of the oblique waveguides. The energy flow for the $q_{3}$ and the $t_{3}$ configurations is observed to be significantly different, i.e., photonic crystal structures exhibit a high sensitivity to geometric parameters as well as dielectric materials.

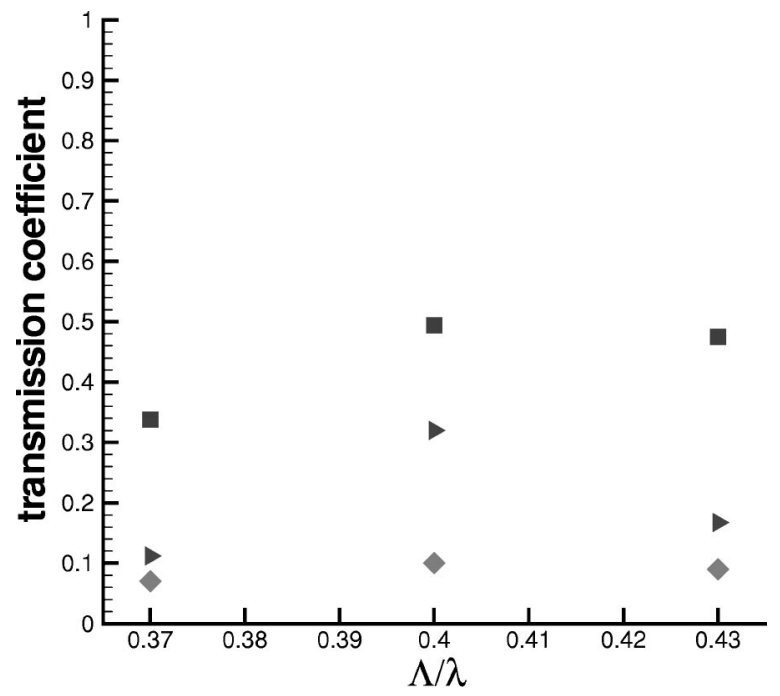

FIG. 14. Single channel waveguide-transmission coefficients for the $t_{2}$ and $t_{3}$ type of branching points with triangular lattices for $W=1.332 \Lambda$ for three normalized frequencies in the defect band. The fractions of incident power that are coupled into the oblique channels above and below the horizontal waveguide are equal because of symmetry. The data represented by squares are for a single oblique channel waveguide in the $t_{2}$ case. Diamonds represent data for one of the oblique channels in the $t_{3}$ case, and left arrows represent data for the horizontal channel waveguide in the $t_{3}$ case. 


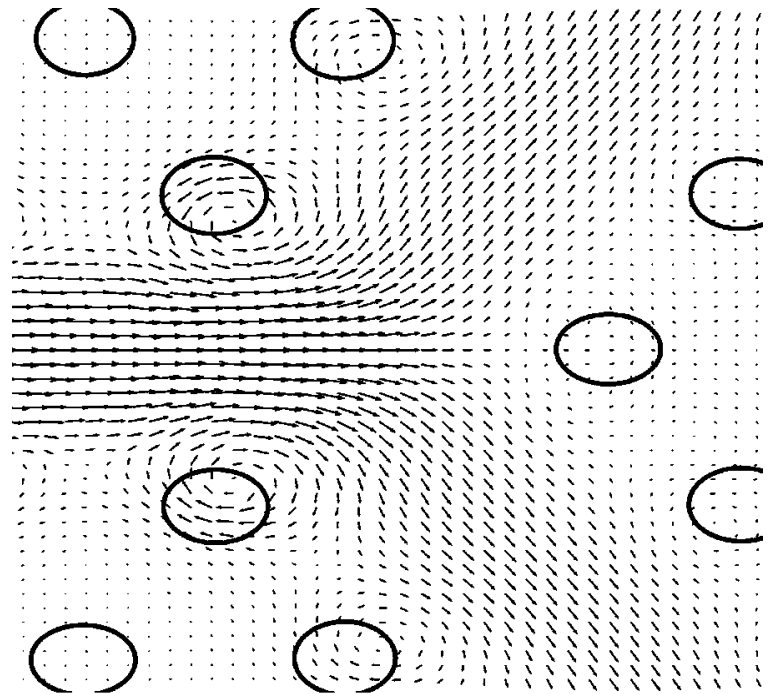

(a)

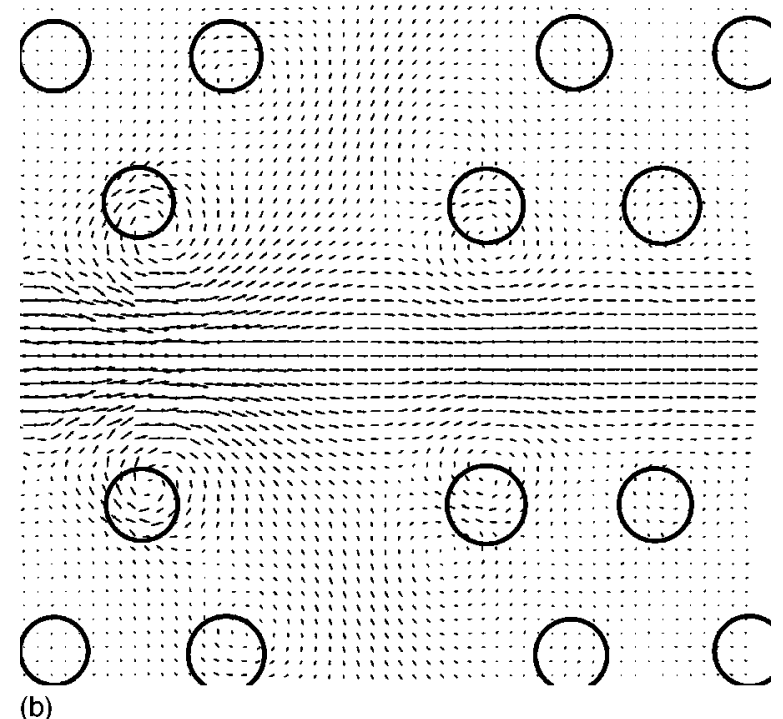

FIG. 15. Energy flow in branching points of type $t_{2}$ and $t_{3}$ for a triangular lattice with $\Lambda / \lambda=0.4$ and $W=1.332 \Lambda$.

\section{CONCLUSION}

Theoretical and numerical investigations of energy flow in photonic crystal waveguides made of line defects in lattices of GaAs rods in a background of air have been presented. The fundamental difference in the electrodynamics of symmetric conventional planar slab waveguides and photonic crystal waveguides has been studied. In the former, energy flows primarily in the direction along the waveguide with a Gaussian distribution of energy across it, whereas in a photonic crystal waveguide the energy flow profile depends on the wavevector and the frequency of the guided mode in the defect band. It was shown that vortices of energy flow may occur, and the net energy flow along the line defect was described via the effective energy propagation velocity (which equals the group velocity in the case of a perfect dielectric).

It was shown that individual modes in multimode waveguides exhibit standing wave patterns across the waveguide section much like those of highly reflective parallelplate microwave metallic guides. Computations of velocity of effective energy flow in photonic crystal waveguides with line defects in triangular lattices have been performed for single-mode and multimode waveguides. Both strong positive, strong negative, and zero dispersion are possible.

The dispersion relations have been calculated for photonic crystal waveguides with two line defect orientations in triangular lattices for different waveguide widths. It was shown that these relations depend strongly on the line-defect orientation in the photonic crystal. Furthermore, a study of energy flow in sharp bends and branching points in the subwavelength scale in photonic crystal waveguides show that although radiation losses are prohibited by the band gap of the cladding, branching points with multiwaveguide connections must be designed carefully in order to minimize backward reflections, and in order to obtain equal distribution of power, as energy flow is highly dependent on the geometric parameters as well as the dielectric materials. These parameters are the nature of the lattice, the orientation of the defect in the photonic crystal, the width of the connecting waveguides, as well as the geometry of the branching points. In general, the width of connecting waveguides must be matched in order to avoid mismatch of the dispersion relations, and resonant branching point modes must match all waveguide modes and respect their symmetry. Photonic crystal waveguides of definite length might enable efficient dispersion compensation, sensing, filtering, wavelength division multiplexing, as well as efficient energy transport around sharp corners because radiation losses are prohibited by the band gap of the cladding, and because of the ease with which they can be tailored to new functionalities simply by creating defects and mode matching by adding or removing geometries with specific material distributions. However, these structures require precise design and refined fabrication techniques as their electrodynamics are highly dependent on geometric parameters. The investigations presented in this paper have been for two-dimensional problems. However, the three-dimensional nature of light propagation in photonic crystals must be taken into account in order to obtain a realistic picture of the diffraction physics, and in order to perform optimal design. More accurate and faster modeling tools in the frequency domain as well as in the time domain must be developed in order to facilitate these tasks.
*Fax: +45 459365 81. Electronic address:

ts@com.dtu.dk

†Electronic address: kim.dridi@risoe.dk

${ }^{1}$ E. Yablonovitch, Phys. Rev. Lett. 58, 2059 (1987).

${ }^{2}$ J.D. Joannopoulos, R.D. Meade, and J.N. Winn, Photonic Crystals (Princeton University Press, Princeton, NJ, 1995).

${ }^{3}$ T.F. Krauss and R.M. De La Rue, Prog. Quantum Electron. 23, 51 (1999)
${ }^{4}$ D. Labilloy, H. Benisty, C. Weisbuch, T.F. Krauss, R.M. De La Rue, V. Bardinal, R. Houdre, U. Oesterle, D. Cassagne, and C. Jouanin, Phys. Rev. Lett. 79, 4147 (1997).

${ }^{5}$ E. Yablonovitch, J. Opt. Soc. Am. B 10, 283 (1993).

${ }^{6}$ M. Plihal and A.A. Maradudin, Phys. Rev. B 44, 8565 (1991).

${ }^{7}$ K. Busch and S. John, Phys. Rev. E 58, 3896 (1998).

${ }^{8}$ P.R. Villeneuve, S. Fan, and J.D. Joannopoulos, Phys. Rev. B 54, 7837 (1996). 
${ }^{9}$ E. Centeno and D. Felbacq, Opt. Commun. 160, 57 (1999).

${ }^{10}$ E. Centeno, B. Guizal, and D. Felbacq, Pure Appl. Opt. 1, L10 (1999).

${ }^{11}$ H. Benisty, J. Appl. Phys. 79, 7483 (1996).

${ }^{12}$ K.H. Dridi and A. Bjarklev, J. Appl. Opt. 38, 1668 (1999).

${ }^{13}$ S.G. Johnson, S. Fan, P.R. Villeneuve, and J.D. Joannopoulos, Phys. Rev. B 60, 5751 (1999).

${ }^{14}$ A. Mekis, S. Fan, and J.D. Joannopoulos, Phys. Rev. B 58, 4809 (1998).

${ }^{15}$ H. A. Haus, Waves and Fields in Optoelectronics (Prentice-Hall, Englewood Cliffs, NJ, 1984).

${ }^{16}$ A. Mekis, J.C. Chen, I. Kurland, S. Fan, P.R. Villeneuve, and J.D.
Joannopoulos, Phys. Rev. Lett. 77, 3787 (1996).

${ }^{17}$ C. Manolatou, S.G. Johnson, S. Fan, P.R. Villeneuve, H.A. Haus, and J.D. Joannopoulos, IEEE J. Lightwave Techno. 17, 1682 (1999).

${ }^{18}$ R.D. Meade, A.M. Rappe, K.D. Brommer, J.D. Joannopoulos, and O.L. Alerhand, Phys. Rev. B 48, 8434 (1993).

${ }^{19}$ M.P. Teter, M.C. Payne, and D.C. Allan, Phys. Rev. B 40, 12255 (1989).

${ }^{20}$ R.D. Meade, A.M. Rappe, K.D. Brommer, J.D. Joannopoulos, and O.L. Alerhand, Phys. Rev. B 55, 15942 (1997).

${ }^{21}$ S. Ramo, J.R. Whinnery, and T. Van Duzer, Fields and Waves in Communication Electronics, 3rd ed. (Wiley, New York, 1994). 